ORI GI NAL

\title{
ESTRUCTURA POBLACIONAL Y ETOLOGÍA DE Bradypus variegatus EN FRAGMENTO DE BOSQUE SECO TROPICAL, CÓRDOBA - COLOMBIA
}

\author{
POPULATIONAL COMPOSITION AND ETHOLOGY OF BradypuS \\ variegatus I N FRAGMENT OF TROPICAL DRY FOREST, \\ CORDOBA - COLOMBIA
}

Jesús Ballesteros C¹, M.Sc, Katia Reyes C², Biól, Javier Racero C³, Esp.

\begin{abstract}
IUniversidad de Córdoba. Facultad de Ciencias Básicas e Ingenierías. Grupo Investigación

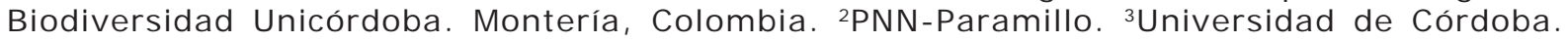
Programa de Maestría en Ciencias Ambientales. Grupo de investigación biodiversidad Unicórdoba.*Correspondencia: jballescor@yahoo.com
\end{abstract}

Recibido: Septiembre 20 de 2008; Aceptado: Julio 30 de 2009

\section{RESUMEN}

Objetivo. Contribuir al conocimiento de la composición poblacional y etología del oso perezoso (Bradypus variegatus) en la reserva natural viento solar, un fragmento de bosque seco tropical (bs-T) del municipio de Moñitos, departamento de Córdoba. Materiales y métodos. Durante octubre 2004 a mayo 2005, se realizaron conteos directos con observación visual en el sendero principal de la reserva, haciendo transectos de un kilómetro de largo x $100 \mathrm{~m}$ de ancho. Se registró el número de animales observados, hábitos alimenticios, estructura poblacional según sexo mediante marcado natural. Se realizaron observaciones sobre comportamiento animal y actividades de desplazamiento diurno, con anotaciones sobre su ecología, y aspectos importantes para la conservación. Resultados. En enero del 2005 se registraron 70 individuos, con una composición de $31 \%$ machos, $53 \%$ hembras y $16 \%$ crías. Durante la época seca del año, (enero-abril) la mayoría de la vegetación arbórea es caducifolia, disminuyendo en forma importante el follaje de los árboles, lo cual facilitó el conteo de la población. La vegetación de bs-T está dominada por Pseudobombax septenatum, cuyo follaje es muy apetecido por el oso perezoso. Conclusiones. Los datos indican que la composición poblacional del oso perezoso en la reserva natural viento solar, está conformada principalmente por individuos adultos, encontrándose una baja proporción de crías; lo que podría estar reflejando problemas de crecimiento poblacional por efecto de baja disponibilidad y baja calidad del hábitat.

Palabras clave: Bradypus variegatus, etología, ecología de poblaciones, hábitos alimenticios, bosque seco tropical. 


\section{ABSTRACT}

Objective. To contribute to the knowledge of the population composition and etology of the lazy bear (Bradypus variegates) in the Natural Reserve Viento Solar, fragment tropical dry forest in Municipality of Moñitos, Department of Córdoba. Materials and methods. During October of 2004 and May of 2005, direct counts with visual observation were carried out in the main path of the reserve, transects of one kilometer of long x $100 \mathrm{~m}$ of wide were done. It was registered the number of observed animals, nutritious habits for visual registration, composition for sex by means of marked natural and they were carried out observations on the animal behavior. Observations of behavior and activities of displacement were made on day, with annotations on their ecology, and important aspects for the conservation. Results. In the month of J anuary, 70 individuals registered, with a composition of $31 \%$ males, $53 \%$ females and $16 \%$ breeding. During the dry time of the year, (J anuaryApril) most of the arboreal vegetation deciduous leaf, diminishing in important form the foliage of the trees, that it facilitated the population's count. The vegetation of tropical dry forest is dominated by Pseudobombax septenatum whose foliage is very felt like by the lazy bear. Conclusions. The data showed that the population composition of the species in the natural reserve is mainly conform by mature individuals, with a low proportion of breeding, that it could be reflecting problems of population growth it likely to readiness and poor habitat quality.

Key words: Bradypus variegatus, ethology, population ecology, eating, tropical dry forest.

\section{NTRODUCCIÓN}

El oso perezoso de tres dedos (Bradypus variegatus) es una de las especies más representativas de la familia Bradypodidae. La especie se distribuye desde Honduras en Centroamérica hasta Suramérica, encontrándose en las costas del Ecuador, a través de Colombia y Venezuela (excepto en Llanos y delta del Río Orinoco), continuando además por las áreas forestales de Ecuador, Perú, Bolivia, por todo Brasil y extendiéndose a la porción del Norte de Argentina (1). Utiliza una gran variedad de hábitat, en especial zonas altas húmedas y espesas (2), cerca a ríos $(3,4)$, y en selvas abiertas (5). Su dieta la componen principalmente hojas de árboles, retoños y otros follajes (6).

Existen varios estudios acerca de la ecología del oso perezoso en regiones tropicales y subtropicales del continente Americano (2, 5, 7-13); pero en la región Caribe colombiana, no existen trabajos de investigación sobre la especie Bradypus variegatus, y se desconoce su estado actual. Por esto, esta investigación de tipo descriptivo, tiene como objetivo contribuir al conocimiento de la composición poblacional y comportamiento animal del oso perezoso; generando información sobre la ecología de Bradypus variegatus, útil en el diseño y establecimiento de estrategias orientadas hacia el manejo y conservación de la especie en aquellas áreas naturales donde ha desaparecido.

\section{MATERI ALES Y MÉTODOS}

Sitio de estudio. La investigación se realizó en la reserva natural viento solar (RNVS), Corregimiento Río Cedro, municipio de Moñitos, localizado en las coordenadas 0758670 latitud norte y 1502528 longitud oeste (Figura 1). La reserva con una extensión de 40 ha, con un alto grado de intervención antrópica, se encuentra dentro de zona de vida de bosque seco tropical, cuya vegetación de cerros y colinas está formada por pastizales, rastrojos y un relicto de bosque seco tropical (bs-T) discontinuo, con árboles caducifolios, y un dosel de 15$20 \mathrm{~m}$ de altura. El área se caracteriza por una época de lluvias (abril-noviembre) y una época seca (diciembre-marzo). El clima es 


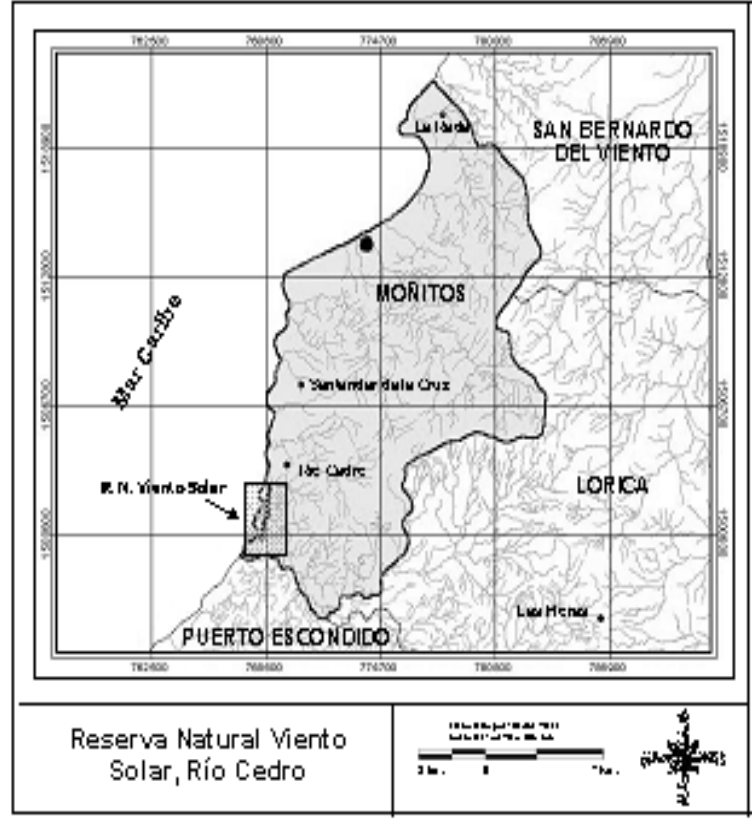

Figura 1. Localización geográfica Reserva Natural Viento Solar, Río Cedro, Moñitos, Departamento de Córdoba-Colombia.

cálido húmedo, con temperatura promedio anual de $27.5^{\circ} \mathrm{C}$, precipitación de $1456 \mathrm{~mm} /$ año y humedad relativa de $88 \%$. El área cuenta con un sendero principal de $3 \mathrm{~km}$ con cobertura boscosa lineal, y una red de senderos peatonales al interior del bosque. Varias fuentes de agua circulan por el bosque: quebrada la Vega, río Cedro, río Mangle, caño del Tigre, cañito del Gobierno, caño Candé, La Isidra, que terminan en un bosque de manglar compuesto por Conocarpus erectus y Rhizophora mangle.

Trabajo de campo. Entre octubre 2004 y mayo 2005 se implementó el método de conteo directo o captura visual de osos perezosos (Bradypus variegatus). Para cuantificar la población se tomó las variables tiempo y espacio, recorriendo el sendero principal durante una hora, siguiendo la metodología de Mayr et al (14). Se determinó la abundancia de la especie según recomendaciones de Brower et al (15). El estudio poblacional se realizó durante ocho meses, en seis salidas de campo con una duración de ocho días continuos cada salida. Se realizaron tres salidas en periodo de lluvia y tres en periodo de sequía. Se realizaron recorridos diurnos y nocturnos, identificando y registrando el momento de mayor actividad del oso perezoso. Se tomaron registros fotográficos, siguiendo las técnicas sugeridas por Brower et al (15).
Densidad y estructura poblacional. La determinación de la estructura poblacional según sexo, se hizo por reconocimiento del marcado natural (dimorfismo sexual). Para la determinación de la proporción machoshembras y la distribución etárea de la población se siguió las recomendaciones de Britton (2). Para el cálculo de la densidad poblacional se tuvo en cuenta el número total de individuos, y el área disponible para la población, determinada mediante polígonos calculados con el programa ArcView 3.3 (16).

Etología. Para el estudio del comportamiento animal se definieron cuatro puntos de observación, teniendo en cuenta la cobertura vegetal, zonas de oferta alimenticia y estado de conservación del hábitat. Se hizo seguimiento de individuos durante las horas luz. En ésta etapa se registró el tiempo de sueño, momentos de alimentación, horas de mayor actividad, tiempo de siesta o descanso, frecuencia, duración y sitio utilizado para el proceso de defecación, y árboles de mayor frecuencia de estadía.

Hábitos alimenticios. La identificación de los hábitos alimenticios se obtuvo por registro visual, identificando las diferentes especies vegetales consumidas. Además, se determinó la oferta natural de alimento disponible, con la participación de miembros de la comunidad local, teniendo en cuenta los conocimientos de éstos sobre la especie. Se identificó taxonómicamente las especies vegetales, mediante levantamientos de vegetación en transectos de $50 \times 2 \mathrm{~m}$, que permitió obtener muestras de la vegetación de lugares de refugio y hábitat utilizados por el oso perezoso.

Relación biológico-social. Para la descripción de la relación biológico-social de la especie, se tuvo en cuenta información primaria obtenida a través de encuestas abiertas aplicadas a la comunidad residente, acogiendo las experiencias vivenciadas de la población con el oso perezoso. Estas encuestas se aplicaron a personas con edades comprendidas entre 13-93 años, quienes tuvieron alguna relación o experiencias con el oso perezoso en la región. 


\section{RESULTADOS}

Durante octubre 2004 a mayo 2005, se registró la presencia de 70 especímenes de Bradypus variegatus, con una relación sexual machohembra de $1: 1,7$. La densidad ecológica fue de 1.75 individuos/ha. Según observaciones de campo y los datos de la encuesta aplicada, el mayor número de partos se presentan en los meses de diciembre y enero.

La composición etárea de la población de osos perezosos en la reserva natural viento solar, refleja que el $58 \%$ de la población está conformada por individuos adultos, $26 \%$ juveniles y $16 \%$ crías (Figura 2).

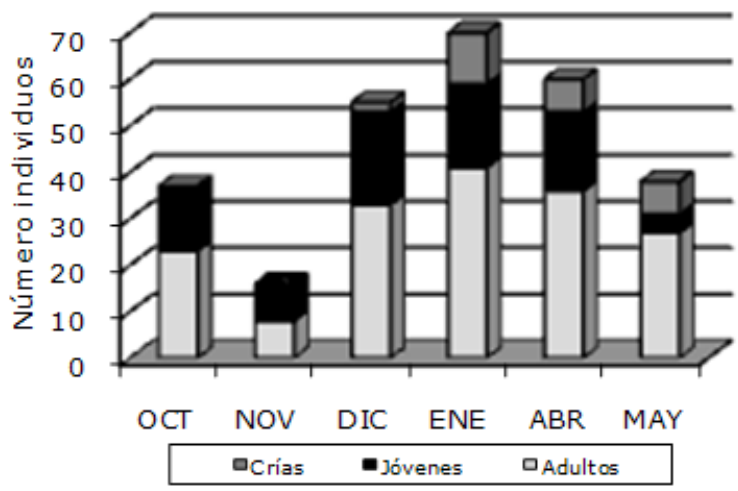

Figura 2. Número de individuos observados durante el tiempo de estudio en la reserva natural viento solar, río CedroMoñitos durante 2004-2005.

La población de perezosos en este relicto de bosque presenta una distribución por sexos de 1: 1,7 machos-hembras, dato que corresponde al $84 \%$ de la población total adulta censada. El $16 \%$ de la población restante corresponde a las crías, con sexo indeterminado (Figura 3).
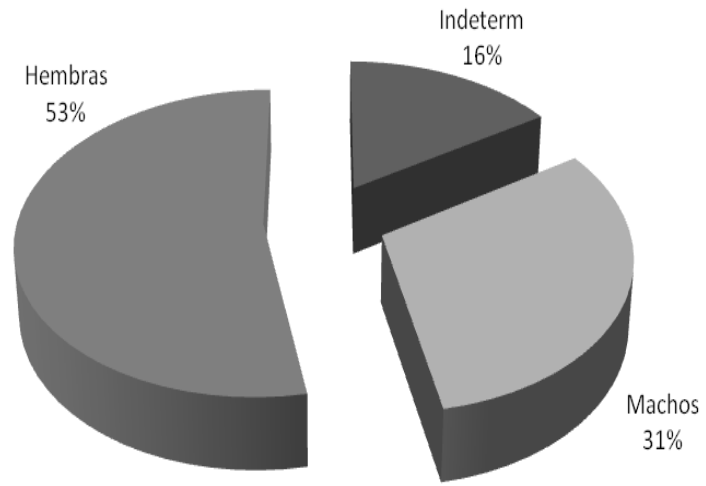

Figura 3. Estructura de la población según sexo del oso perezoso (Bradypus variegatus) en la reserva natural viento solar, durante 2004-2005.

Durante el estudio se encontró que la población de perezosos se distribuye en toda el área disponible de la reserva y sus alrededores. Según información primaria obtenida en campo, información secundaria encontrada, y la obtenida de los pobladores residentes en la zona, la presencia de osos perezosos es muy común y están distribuidos en toda el área del Corregimiento Río Cedro; pero la mayor abundancia se presenta en zona litoral a orillas de la playa.

Observaciones de campo confirman que los osos perezosos tres dedos, en la RNVS presentan hábitos diurnos y nocturnos. Al iniciar el día se observó que la especie acostumbra a posicionarse en lo más alto de las ramas de árboles de Pseudobombax septenatum (bonga). Estos animales son de hábitos solitarios (Figura 4); sin embargo, en algunas ocasiones durante los censos realizados, se registraron 14 grupos integrados por tres a cuatro individuos,
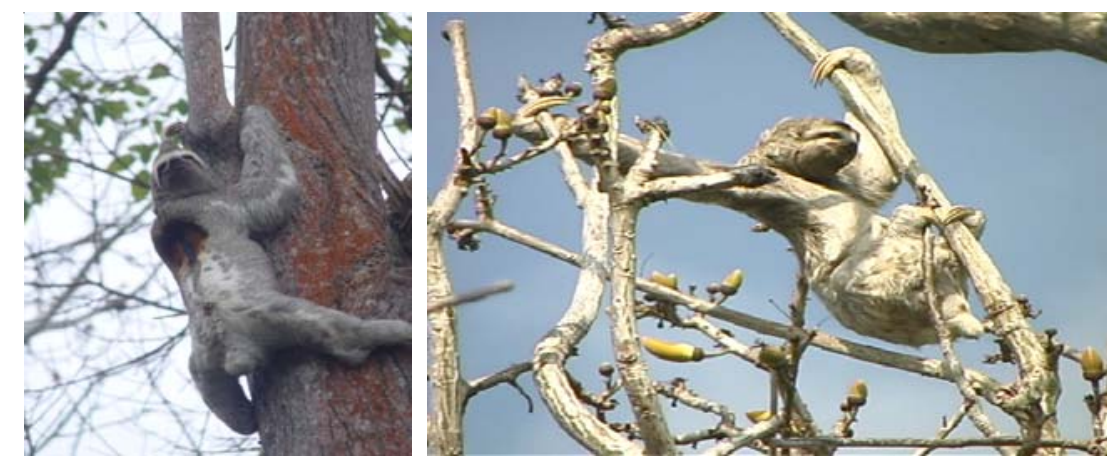

Figura 4. Aspecto general del oso perezoso (Bradypus variegatus) trepando un árbol de bonga, y ubicándose en las ramas más altas. Obsérvese la franja de color naranja sobre el lomo, característica de los machos adultos. 
generalmente compuestos por un macho y 2-3 hembras. La rutina de acicalamiento fue observada en horas de la tarde (17:00 horas) con una duración de 30 minutos, conducta registrada en $19 \%$ de los individuos observados.

El hábitat del oso perezoso en la RNVS está dominada en gran parte por árboles de bonga (Pseudobombax septenatum) y jobos (Spondias mombin), vegetación típica de bs$\mathrm{T}$ costanero. El perezoso se alimenta principalmente de retoños, botones florales de Pseudobombax septenatum, Cecropia sp. $y$ en algunos casos, de frutos de jobo (Spondias mombin) y uva de playa (Coccoloba uvifera), entre otras. Pseudobombax septenatum es la especie arbórea más abundante a lo largo del área de estudio, y es la principal fuente de alimento para el oso perezoso, debido fundamentalmente a que ofrece alimento durante todo el año, y del cual consume sus hojas más nuevas, botones florales y flores.

Se observó una ampliación de la dieta del perezoso a otras especies vegetales, entre las cuales están el Oyeto (Lecytis minor), jobo (Spondias mombin), guácimo (Guazuma ulmifolia), ceiba (Bombacopsis quinata), almácigo (Bursera simarouba), roble (Tabebuia rosea) y el guarumo (Cecropia sp.). Sin embargo, los perezosos nunca dejaron de alimentarse de la bonga al inicio del periodo seco (diciembre), cuando éstos árboles empiezan a desfoliarse, estrategia ecofisiológica adaptativa durante la época seca del año.

El principal factor de mortalidad del oso perezoso en el área de estudio está relacionada con la caída de animales desde grandes alturas, especialmente durante la época de los vientos alisios (diciembre hasta mediados de abril); siendo también, la depredación por perros domésticos (Canis familiaris) que atacan a los perezosos cuando descienden de los árboles.

\section{DISCUSIÓN}

Los datos poblacionales encontrados, reflejan en el hecho de encontrar la mayor proporción de crías en enero, que coincide con el inicio de la época seca del año, cuando se presenta la defoliación de los árboles, y finalización del periodo de gestación de las hembras. Se encontró que las crías están al cuidado materno durante los meses de diciembre a mayo, lo cual concuerda con lo descrito por Oliveira (17), quien describe que las hembras cargan a sus hijos durante aproximadamente los primeros nueve meses de vida.

La estructura poblacional encontrada, probablemente indica poco éxito de crianza; igualmente el bajo porcentaje de población juvenil, puede ser otro indicativo de que las poblaciones no se están manteniendo en equilibrio poblacional debido a efectos ambientales, así como por la baja oferta de hábitat y alimento. Sin embargo, hay que considerar que la proporción de edades no puede interpretarse sin un conocimiento del ritmo de crecimiento de la especie (18).

La abundancia poblacional de perezosos en esta área se debe fundamentalmente a que disponen de una importante cobertura vegetal, oferta de alimento, refugio y que en esta área se presenta la mayor diversidad de especies arbóreas que frecuentan los osos perezosos. Todas estas características del hábitat, favorecen la presencia y conservación del oso perezoso tres dedos. La tendencia a posicionarse en lo más alto de las ramas de árboles, probablemente es una respuesta de comportamiento para elevar la temperatura corporal y el metabolismo por exposición a la radiación solar, como lo reporta McNab (19). Luego de cuatro o cinco horas de exposición al sol, buscan refugio entre las ramas con abundantes hojas, lo que permiten la regulación de la temperatura, ayudando al proceso de digestión y, a la vez, como refugio para protegerse de los depredadores.

El registro de grupos de perezosos con 3-4 individuos, nos indica una interacción social intraespecífica que coincide con lo descrito por Polanco (20), quien registró 445 encuentros con perezosos, de los cuales un $10 \%$ fueron grupos de dos o más individuos en un mismo árbol, y de éstos, tres correspondían a una hembra con su cría. Durante el trabajo de campo se observó 
comportamientos de agresión y lucha por parte de machos en defensa de su territorio. Se encontró que la especie presenta poca actividad durante el día, lo que puede ser una forma de ahorrar energía para los recorridos en las noches, que suelen ser frías. Sin embargo, invierten buena parte de su tiempo en actividades de acicalamiento, especialmente en horas de la tarde.

En relación con la reproducción, durante la época de apareamiento se registraron silbidos unitonales ( $<<a-$ ee $>>$ ) emitidos por las hembras para llamar a los machos. El tiempo de cortejo y persecución a las hembras por los árboles por parte de los machos puede durar de 30 a 90 minutos, y luego del apareamiento, las parejas permanecen juntas hasta dos días después. El tiempo de gestación de las hembras en la zona fue de seis meses, dando a luz colgadas de los árboles, una cría por parto, lo cual coincide con los datos descritos por Cabrera y Yépes (21).

El hábitat del oso perezoso en esta región, el cual está dominado por Pseudobombax septenatum y Spondias mombin, concuerda con las descripciones realizadas por Mongomery y Sunquist (9). Esta preferencia se debe probablemente a que estos árboles tienen la copa expuesta al sol. Aunque el área de estudio no presenta áreas boscosas grandes (bosques estratificados), una franja de vegetación boscosa de dos km, aloja la mayor cantidad de perezosos en el sendero ecológico de la RNVS; pero en el tramo conocido como La Vega, un sitio donde se presenta la mayor cobertura vegetal, se encontró la mayor densidad de población de perezosos.

Siendo Pseudobombax septenatum la especie arbórea más abundante y la principal fuente de alimento para los perezosos durante la mayor parte del año, éste árbol representa gran importancia ecológica; lo cual es reconocido por el $42 \%$ de los pobladores locales encuestados. Estas observaciones llevan a considerar a la bonga como una especie clave a la hora de realizar planes de conservación y reforestación, además de otras especies nativas presentes; lo que puede propiciar hábitat adecuados para la conservación de la población del oso perezoso y otros animales.

Las observaciones realizadas con relación a la diversidad en la dieta del perezoso, demuestra que los osos perezosos tienen una dieta variada de plantas arbóreas que pueden consumir en distintas épocas del año (Lecytis minor, Spondias mombin, Guazuma ulmifolia, Bombacopsis quinata, Bursera simarouba, Tabebuia rosea, Cecropia sp., entre otras). Se confirma el gusto del los osos perezosos por Pseudobombax septenatum y Cecropia sp, sugerido por otros autores $(7,9,20)$, y Spondias mombin, Guazuma ulmifolia, Coccoloba uvifera con más frecuencia que otras, debido probablemente a la amplia distribución de estas especies arbóreas en el hábitat disponible.

La principal amenaza para la conservación de la especie Bradypus variegatus en la RNVS, está relacionada con la depredación por perros domésticos cazadores (Canis familiaris) que persiguen y matan a los perezosos cuando descienden de los árboles. En algunas ocasiones se han observado algunas aves rapaces que atacan a las crías. Es importante mencionar que la conservación de la especie en la zona se ha visto favorecida, debido a que se ha logrado ampliar las alternativas de producción y alimentación para la comunidad local, especialmente en actividades de la pesca artesanal, cultivos agrícolas, y actividades ecoturísticas adelantadas por la RNVS, disminuyendo así la presión sobre la especie por los residentes.

En la relación biológico-social, las actividades antrópicas están relacionadas directamente con aquellas que alteran el medio natural, aunque no se encontró amenaza directa del hombre para la conservación del perezoso. Es importante tener en cuenta el poco conocimiento que por parte de los habitantes locales tienen de la importancia ecológica, situación que plantea una amenaza potencial para la conservación del hábitat y Bradypus variegatus, junto con otras especies silvestres. Los bajos niveles de escolaridad y el analfabetismo presente en la región, puede tener un efecto negativo en la conservación de la biodiversidad regional. 
En conclusión, la población de Bradypus variegatus en la RNVS con el $58 \%$ de la población adulta y baja proporción de crías, puede ser un indicativo de un bajo éxito reproductivo, en respuesta a deficiente oferta de alimento y baja calidad del hábitat; situación que podría disminuir la población. En el hábitat disponible en la RNVS, Pseudobombax septenatum representa la principal fuente de alimento, que por su abundancia en el área de estudio, podría considerarse como especie clave para la conservación del perezoso; pero durante el periodo seco del año, cuando la bonga deja caer sus hojas, su dieta se amplía a otras especies vegetales, entre las que están las especies Spondias mombin, Guazuma ulmifolia, Bombacopsis quinata y Bursera simarouba.

La especie Bradypus variegatus no presenta amenazas antrópicas directas para su conservación, pero es importante realizar trabajos a nivel poblacional en los alrededores de la reserva para determinar el estado actual de la especie, pues, la actitud de la población humana local y las condiciones ambientales del hábitat no son las más adecuadas para asegurar la conservación de la especie.

\section{Agradecimientos}

Los autores expresan sus agradecimientos a la Fundación Viento solar, por el apoyo logístico para realizar la investigación en la Reserva Natural Viento Solar. A Carlos Vidal y a los habitantes del Corregimiento Río Cedro por su colaboración en la investigación y trabajo de campo. A la Universidad de Córdoba, por el apoyo logístico y financiero de la investigación a través de la Oficina de Investigación y Extensión.

\section{REFERENCIAS}

1. Cabrera A. Catálogo de los Mamíferos de América del Sur. Rev Mus Argentino Cienc Nat 1958; 4: 1-307.

2. Britton S.W. Form and function in the sloth. Q Rev Biol 1941; 16: 14-34.

3. Goldman E.A. Mammals of Panama. Smithsonian Misc Coll 1920; 69(5): 1309.

4. Beebe W. The Three-Toed Sloth. Zoologica. 1926; 26: 3-66.

5. Menegaux A. Sur le squelette du member anterior de Bradypus torquatus. C. R. Acad. Sci. III. 1908; 147: 637-640.

6. Bourliere F. The natural history of mammals. Londres: Harrap; 1955.

7. Eisenberg J, Thorington R.J r. A Preliminary Analisis of a Neotropical Mammal Fauna. Biotropica 1973; 5(3): 150-161.
8. Montgomery G.G, Sunquist M.E. Impact of sloths on Neotropical forest energy flow and nutrient cycling. In the tropical ecolological systems (letter). New York: Sprint-veday. Ed. F. B. Golley and E. Medina; 1975.

9. Montgomery G.G, Sunquist M.E. Habitat selection and use by two-toed and three-toed sloths. In the Ecology of arboreal folivores (letter), Ed. G.G. Montgomery. Washington, D.C: Smithsonian Institution Press; 1978.

10. Goffart M. Function and form in the sloth. Oxford: Pergamon Press; 1971.

11. Muttenz G.S. Manual de Identificación. Convención sobre el Comercio Internacional de Especies Amenazadas de Fauna y Flora Silvestre. Ginebra. Suiza: Secret. CITES; 1987.

12. Krieg H. Das Verhalten der Gürteltiere (Dasypodidae). In Handbuch der Zoologie (letter). W. Kuekenthal and T. Krubach, Eds, 1961; 24-31 pp. 
13. Tirler $\mathrm{H}$. A sloth in the family. New York: Walker and company Ed; 1966.

14. Mayr J., Martínez C., Cifuentes M., Andrade A., Gómez D., Gutiérrez F., Montenegro M., Moreno R., Reina R., Rivera A., Rodríguez C., y Vaca D. Guía para la Evaluación de Poblaciones de Fauna Silvestre. Bogotá: Ministerio del medio ambiente; 2000.

15. Brower J.E., Zar J.H., Ende C.N. Field and laboratory methods for general ecology. USA: Wm. C. Brown Publishers; 1989.

16. Arc View 3.3. Environmental Systems Research Institute. Inc. New Delhi: India; 2002.

17. Oliveira V.L. Reabilitaçao e manutençao dos bichos-preguiça (Bradypus torquatus e B. variegatus - mammalia, Bradypodidae) em semi cativeiro. Reserva zoobotánica (Martinha). 1999.
18. Caughley G. Interpretation of age ratios. J Wildl Manage 1974a; 38(3): 557-562.

19. McNab B. Energetics of arboreal folivores: physiological problems and ecological consequences of feeding on a ubiquitous food supply. In the Ecology of arboreal folivores (letter), ed. G.G. Montgomery. Washington D.C: Smithsonian Institution Press; 1978.

20. Polanco R. Estrategias reproductivas y estimación poblacional en Bradypus variegatus. [Tesis de Maestría]. Costa Rica: Universidad Nacional, Facultad de ciencias Básicas; 1998.

21. Cabrera A, Yépes J. Mamíferos de Sur América. Historia Natural. Buenos Aires. Compañía Argentina de Editores (EDIAR S.A). Tomo II. 1960; 56-62 pp. 